\title{
Validación de un instrumento para valorar la percepción del aprendizaje y el nivel de diversión del alumno en educación física. El semáforo
}

\author{
Validation of an instrument to assess student learning perception \\ and level of enjoyment in physical education. The traffic light
}

\section{Validação de um instrumento para avaliar a percepção da aprendizagem e o nível de diversão de alunos de educação física. O semáforo}

\author{
José Manuel Palao Andrés* y Elena Hernández Hernández** \\ *Universidad Murcia, España \\ **Universidad Pablo de Olavide. Sevilla. España
}

\begin{abstract}
Resumen: El objetivo del presente trabajo fue validar un instrumento de evaluación de la percepción del aprendizaje y el nivel de diversión de los alumnos en clase de educación física ("el semáforo"). El instrumento "el semáforo" constaba de dos cuestiones cerradas con tres posibles respuestas (¿Cuánto crees que has aprendido / te has divertido en esta sesión?), relacionadas con la percepción que el alumno tiene sobre el nivel de aprendizaje, y el nivel de diversión alcanzado durante la clase de educación física. Los alumnos para responder deben marcar el color con el que se identificaba (rojo=poco, amarillo = algo, o verde=mucho) en función de su percepción . Se realizó una validación del contenido a través del acuerdo y consenso de diez jueces expertos Los resultados indicaron que el cuestionario tuvo por parte de los jueces expertos una valoración de 8.70 en la pregunta de percepción del aprendizaje y 8.80 en la pregunta de percepción de la diversión. A partir de los resultados obtenidos se pueden establecer las siguientes conclusiones: el cuestionario "El semáforo" dispone de niveles de validez de contenido para evaluar la percepción del alumno sobre el nivel de aprendizaje y el grado de diversión alcanzado de iniciación en las clases de educación física. El instrumento diseńado, por ser rápido y fácil cumplimiento, es apropiado para ser utilizado en el ámbito educativo reglado.

Palabras claves: test, evaluación, enseñanza reglada, alumno,

Abstract: The aim of this study was to validate an instrument for assessing students' learning perception and enjoyment in physical education class (the "traffic light"). The instrument of the "traffic light" consisted of two closed questions with three possible answers (How much have you learned? / How much have you enjoyed class?) related to students learning perception and enjoyment in physical education class. Students answer by marking the color that identified their perceptions of learning and enjoyment during the class (red $=$ little, yellow $=$ some, or green $=$ much). Validation of
\end{abstract}

\section{Introducción}

La educación física en la etapa de Educación Secundaria Obligatoria debe contribuir a la formación integral y al desarrollo de los alumnos. Las clases de educación física en esta

Universidad Pablo Olavide de Sevilla

Departamento de Deporte e informática, edificio 14, planta 4a ${ }^{\mathrm{a}}$, despacho 45. Carretera de Utrera, km. 1. Sevilla, 41013. the content through agreement and consensus of 10 expert judges. The expert judges gave the question of learning perception an assessment of 8.70 and the question of enjoyment perception an assessment of 8.80 . Through the results obtained, the following conclusion can be established: the "traffic light" questionnaire has content validity for evaluating the student's perception of learning and enjoyment in physical education classes. The instrument is appropriate for use in formal educational (it is fast and easy). Keywords: test, evaluation, formal education, student

Resumo: O objetivo do presente trabalho foi de validar um instrumento de avaliação da percepção da aprendizagem e o nível de diversão dos alunos na classe de educação física (o semáforo). O instrumento "semáforo" constava de duas questôes fechadas com três possíveis respostas (Quanto acredita ter aprendido? / Você se divertiu com essa sessão?), relacionadas com a percepção que o aluno tem sobre o nível de aprendizagem e o nível de diversão alcançada durante a aula de educaçáo física. Os alunos tiveram que marcar a cor que identificava (vermelho=pouco, amarelo=algo e verde muito) em função de sua própria percepção. Foi realizada uma validação do conteúdo através do acordo e consenso de dez juízes. Os resultados indicaram que o questionário teve uma pontuaçáo de 8,70 por parte dos juízes na pergunta de percepçáo de aprendizagem e um 8,80 na pergunta de diversáo. A partir dos resultados obtidos, podem estabelecer as seguintes conclusōes: o questionário "o semáforo" dispóe de níveis de validez de conteúdo para avaliar a percepção de alunos sobre o nível de aprendizagem e o grau de diversão alcançado nas aulas de educação física. O instrumento desenhado por ser rápido e de fácil execuçấo é apropriado para ser utilizado no âmbito educativo com regularidade.

Palavras Chave: teste, avaliação, ensino regular, aluno.

etapa educativa deben de dar respuesta, entre otros objetivos, al fomento de la práctica de actividad física (B.O.E., 2000). El período de edad que responde a esta etapa, coincide con el período en el que se da más abandono de la práctica de actividad física (Casimiro, 1999; Sallis, Mckenzie, y Alcaráz, 1993; Weinberg, y Gould, 1995). Entre los motivos que son atribuidos a este abandono está la influencia que ejerce el pro- 
fesor en el proceso de enseñanza como la figura importante en relación con la motivación del alumno (Ortega, Calderón, Palao, y Puigcerver, 2008; Silverman, 1985; Castejón, Giménez, Jiménez, y López, 2003). Esta preocupación en el ámbito de la investigación educativa es recogida en trabajos como los publicados por Moreno, Vera, y Cervelló (2006). En ellos, los autores destacan como aspectos que mejoran la motivación por las clases de educación física, la cesión de responsabilidad al alumno (Cervelló y cols., 2004; Duda, 2001), y el hecho de provocar que sea el propio alumno el que tome decisiones y revise cómo se está desarrollando el proceso de instrucción (Mc Caughtry y Rovegno, 2003). En este sentido, los juicios que los alumnos realicen a través de procedimientos como la autoevaluación y la co-evaluación, les permitirán obtener una mejor comprensión de los contenidos ya que, para ello, deberán realizar una revisión de los conocimientos proporcionados por el profesor, lo que incidirá de forma positiva en la percepción que tiene el alumno de sus propias competencias, y por tanto, aumentará la satisfacción por el proceso llevado a cabo en clase (Hassandra, Goudas, y Chroni, 2003; Ntounamis, Pensgard, Martín, y Pipe, 2004; Xiang, McBridge, y Guan, 2004).

Los trabajos desarrollados por Siendentop (1998) muestran como la supervisión junto con la implantación de un mecanismo de evaluación son dos elementos de la enseñanza determinantes para aumentar la eficacia de la actuación del profesor en clases de educación física. En este caso, la implantación de mecanismos para responsabilizar a los alumnos durante la evaluación llevará consigo el tener en cuenta aspectos como la organización, la implicación en las tareas de aprendizaje utilizadas, y los intereses mostrados por los alumnos (Moreno, Vera, y Cervelló, 2006). Estos mecanismos provocan que el profesor tenga que reflexionar sobre cuál es la manera más eficaz de plantear las tareas para cederle responsabilidad al alumno en la toma de decisiones dentro del contexto educativo. De este modo, permitir la participación del alumno en la evaluación vendrá determinado por la cesión de responsabilidad que el educador facilita, que a su vez, aumentará el interés que ésta tiene sobre la participación del alumno en clase. No se debe olvidar que este factor, el interés del alumno, deberá estar presente en todo momento, ya que aumenta de forma poderosa la motivación del alumno por las clases de educación física. Es por esto que el objetivo del presente trabajo es el de diseñar un instrumento que permita la participación del alumno durante la evaluación de algunos de los elementos que intervienen en el proceso de enseñanza, desarrollados dentro del contexto educativo.

En el ámbito de la educación física (y la iniciación deportiva) es frecuente encontrar instrumentos que permitan controlar y evaluar aspectos relacionados con el rendimiento del alumno en clase (normalmente desde el punto de vista de la superación o no de contenidos a nivel procedimental, o con- ceptual). En este contexto, es menos frecuente encontrar instrumentos que permitan valorar otros elementos relacionados con las interacciones que se producen en clase entre profesoralumno, y que a su vez, puedan ser utilizados por cualquiera de los agentes educativos. A pesar de ello, existen algunos trabajos que han contribuido a obtener herramientas útiles para que el profesor pueda evaluar aspectos actitudinales y de percepción del alumnado (Ortega, Calderón, Palao, y Puigcerver, 2008), y las preferencias o el nivel de satisfacción durante la iniciación deportiva (Ortega, Jiménez, Palao, Sainz de Baranda, 2008). A través de la técnica del cuestionario, estos instrumentos recogen aspectos que permiten valorar elementos como el divertimento en la práctica deportiva, el nivel de diversión, o la actitud del profesor y de los alumnos en clase. Dentro del ámbito de la educación física, el cuestionario es la herramienta más utilizada para obtener información, debido a que se trata de un instrumento de fácil aplicación que conlleva, a su vez, pérdida de poco tiempo para su aplicación (Silverman y Subramanian, 1999; Thomas y Nelson, 2007). Tomando como referencia estos trabajos, y con el objetivo de crear una herramienta que sea de fácil aplicabilidad en un contexto reglado como las clases de educación física en la Etapa de Educación Secundaria Obligatoria, la finalidad del presente trabajo fue validar un instrumento de evaluación de la percepción del aprendizaje y el nivel de diversión de los alumnos en clase de educación física ("el semáforo").

\section{Método}

\section{Participantes}

Para realizar la validación del instrumento se ha utilizado la técnica de jueces expertos. Para la validez de contenido se emplearon diez jueces expertos. Cinco de ellos poseían el grado de doctor en ciencias de la actividad física y el deporte, de entrenador nacional de un deporte, y tenían al menos cuatro años de experiencia en docencia universitaria. Cinco de ellos eran licenciados en educación física, poseían el título de entrenador nacional de un deporte, y tenían al menos tres ańos de experiencia dando clase en centros de educación secundaria.

\section{Diseño}

Para el estudio de la validez de los cuestionarios se definió la "validez de contenido" como el grado en que una prueba representa de forma adecuada lo que se ha realizado (Thomas, y Nelson, 2007; Wiersma, 2001). Para alcanzar niveles óptimos de validez de contenido, con el instrumento objeto de estudio se utilizó la técnica de jueces expertos. Las variables objeto de estudio para valorar la validez fueron:

a) Validez de contenido mediante jueces expertos. Se so- 
licitó a los jueces expertos que valorasen diferentes aspectos sobre la adecuación de cada cuestión a la etapa educativa a la que iban dirigidas, una valoración conceptual (grado de comprensión, adecuación en la redacción, etc.), y una valoración cuantitativa de los mismos (escala del 1 al 10). Además se les dejó la opción de que anotasen algunas observaciones o alternativas con respecto a algún aspecto relacionado con las preguntas y los ítems de las respuestas.

b) Grado de pertenencia al objeto de estudio. Se registró en qué medida las dos cuestiones planteadas debían formar parte del mismo. Para ello, se solicitó a los jueces expertos que valorasen conceptualmente la importancia de cada una de las preguntas de forma independiente. En este sentido, los jueces expertos indicaban la necesidad de que el ítem formase, o no, parte del instrumento. De igual modo, en una escala de 1 a 10 indicaban el grado de pertenencia de los ítems al instrumento.

c) Grado de adecuación y comprensión. Se registró el grado de comprensión y adecuación en la definición y redacción de cada pregunta.

\section{Instrumento}

El instrumento a validar fue una adaptación del elaborado por Palao y Ruiz (2003). Para ello se tomó como referencia la propuesta del profesor Dr. Delgado-Nogueras (2001) de valorar mediante colores la percepción de los alumnos en clase, y el formato empleado por McGrath, De Veber y Hearn (1985) denominada como "escala de las nueve caras". En éste último el niño tiene que escoger cuál de los nueve rostros dibujados se adapta más a su estado de ánimo. Teniendo en cuenta ambas aportaciones, se diseñó un instrumento que ha sido denominado como "semáforo", por guardar la misma estructura de colores que la de un semáforo. Este instrumento fue empleado para valorar la percepción de los alumnos universitarios a nivel de diversión en clases de iniciación a un deporte (Palao y Ruiz, 2003). La versión original del instrumento primitivo constaba de una pregunta sobre la percepción de la diversión vivida. Este instrumento fue adaptado y empleado para otras investigaciones ańadiéndose una pregunta más para valorar el nivel de aprendizaje (ej. Hernández, Nortes, Leante, y Palao, 2009). Esta versión adaptada es con la que se realizó el proceso de validación. El instrumento del "semáforo" consta de dos cuestiones cerradas con tres posibles respuestas (¿Cuánto crees que has aprendido / te has divertido en esta sesión?), relacionadas con la percepción que el alumno tiene sobre el nivel de aprendizaje, y el nivel de diversión alcanzado durante la clase de educación física. Los alumnos para responder debían marcar el color con el que se identificaba (rojo=poco, amarillo $=$ algo, o verde $=$ mucho) en función de su percepción hacia lo que habían aprendido, o si se habían divertido durante la sesión.

\section{Procedimiento}

El proceso de validación de contenido fue llevado a cabo en tres fases (Carretero-Dios, y Pérez, 2007). En primer lugar, el instrumento fue enviado a un grupo de 15 jueces expertos. Se solicitó a los jueces expertos que valorasen diferentes aspectos sobre la información inicial, la escala de medida, y los preguntas del instrumento, y una valoración global del mismo (Wiersma, 2001). Con respecto al texto, a la escala de medida, y a la valoración global se solicitó a los jueces expertos que hiciesen una valoración cualitativa (grado de comprensión, adecuación en la redacción, pertinencia de las preguntas, etc.). A nivel de valoración global se solicitó a los expertos que realizasen una valoración cuantitativa de las preguntas del instrumento (escala de 1 a 10). A continuación, y a partir de las respuestas de los jueces expertos, se realizó una interpretación de las respuestas de los jueces expertos. Siguiendo la propuesta de Bulger y Housner (2007) se decidió eliminar todos aquellos ítems con valores medios inferiores a 7, modificar los ítems con valores entre 7.1 y 8 , y aceptar los superiores a 8.1. Tras lo cual se modificaron el instrumento de acuerdo con las recomendaciones de los jueces expertos.

\section{Estadística}

El registro y almacenamiento de los datos del instrumento se realizó con una hoja de cálculo Excel 2007 de Microsoft. Para el análisis de los datos se realizó un análisis descriptivo de los datos. Para este análisis se utilizó el paquete informático SPSS 15.0 en el entorno Windows.

\section{Resultados}

Diez de los 15 jueces respondieron a la petición de los investigadores.

En relación a la idoneidad del instrumento con respecto a la etapa para el que fue construido, la valoración de los jueces fue positiva, indicando que eran adecuados para dicho nivel.

En relación con cada una de las preguntas, la valoración de los ítems fue apreciada como adecuada en su formulación y comprensión con respecto al objetivo marcado para el instrumento. Sin embargo, se aprecian aportaciones con respecto a términos, y enunciaciones adecuadas a nivel conceptual. La mayoría de los jueces indicaron la necesidad de realizar pequeñas modificaciones en la redacción de las preguntas para que fuesen aún más simples y directas. Algunas de las sugerencias al respecto fueron: “ ¿Cuánto te has divertido en la sesión?" (Juez 7); “Cuál ha sido tu grado de aprendizaje?” (Juez 5); “Cuánto consideras que has aprendido en la sesión?” (Juez 3); etc.

Otras de las aportaciones realizadas por los expertos fue la posibilidad de incluir en el instrumento la explicación de lo 
que significaba cada uno de los colores con los que tenían que responder, y que a su vez, debía de identificarse con la percepción del alumno. Por ejemplo, el juez 3 propone añadir: mucho (verde), algo (amarillo), y nada (rojo); de la misma forma, el juez 5 propone: bajo (rojo), medio (amarillo), alto (verde).

La valoración global del cuestionario fue positiva. Por ejemplo, el juez 8 destacó la rapidez y sencillez del instrumento para poder ser aplicado en clases de educación física. No obstante, todos los expertos indican la necesidad de ubicar la pregunta a valorar a continuación del enunciado, antes de los dibujos, y no debajo En la tabla 1 se puede observar la valoración numérica del cuestionario. La valoración global fue de 8,75 . Solo se aprecia una valoración más baja de los jueces número 2 y 5 . Estos jueces expertos indicaron que la baja valoración se debía a la necesidad de modificar la pregunta respecto a su redacción, para que ésta fuese mejor entendida por los alumnos que debían de responder al instrumento.

Tabla 1. Valoración de los expertos sobre el instrumento de valoración de la percepción del alumno sobre el aprendizaje y el nivel de diversión en clase.

\begin{tabular}{lccccccccccc}
\hline Expertos & J1 & J2 & J3 & J4 & J5 & J6 & J7 & J8 & J9 & J10 & Val. medio \\
\hline Aprendizaje & 10 & 7 & 8 & 10 & 7 & 9 & 8 & 9 & 9 & 10 & 8.70 \\
Acción & $\mathrm{No}$ & $\mathrm{Si}$ & $\mathrm{No}$ & $\mathrm{No}$ & $\mathrm{Si}$ & $\mathrm{No}$ & $\mathrm{No}$ & $\mathrm{No}$ & No & No & \\
\hline Diversión & 10 & 7 & 8 & 10 & 7 & 9 & 8 & 10 & 9 & 10 & 8.80 \\
Acción & $\mathrm{No}$ & $\mathrm{Si}$ & $\mathrm{No}$ & $\mathrm{No}$ & $\mathrm{Si}$ & $\mathrm{No}$ & $\mathrm{No}$ & $\mathrm{No}$ & No & No & \\
\hline
\end{tabular}

Leyenda: sobre la acción que se realizó, si el valor obtenido era superior a 8 (no), si el valor obtenido era de 7 o superior, el ítem fue revisado y modificado (si); si el valor era inferior a 7, se suprimía o cambiaba por otro (e).

\section{Discusión}

Los jueces expertos realizaron aportaciones para la mejora del instrumento. Estas aportaciones cualitativas giraron en torno a: a) modificar la redacción de la pregunta para focalizar mejor la atención de los solicitado; b) añadir una explicación de con qué grado de respuesta debían identificar los diferentes colores del semáforo; y c) ubicar la cuestión realizada antes del dibujo del semáforo. Estas contribuciones de tipo cualitativo, por parte de los jueces expertos, que ayudan a dar consistencia al instrumento, son indispensables en el desarrollo de un instrumento (Bulger y Housner, 2007; CarreteroDios y Pérez, 2005; Padilla, Gómez, Hidalgo y Muñiz, 2007; Ortega et al., 2008; Subramanian y Silverman, 2000; Wieserma, 2001). Estas observaciones aportan información relevante para eliminar o modificar posibles cuestiones (Dunn, Bouffard y Rogers, 1999). Estas opiniones sugeridas por los jueces expertos fueron tenidas en cuenta y modificadas en el instrumento final (anexos 3 y 4). Por último, los jueces destacaron la sencillez y rapidez de aplicación del instrumento. Estas cualidades le hacen ser una herramienta útil para ser utilizado en el contexto educativo reglado, donde es limitado el tiempo disponible para realizar las clases. El instrumento aquí presentado se puede aplicar dentro de las clases de educación física ya que en un intervalo corto de tiempo, y dentro de la misma dinámica de clase, el alumno puede valorar la clase respondiendo a las dos cuestiones. Tiene dos modalidades que pueden emplearse de forma conjunta, o por separada, en función de los intereses del docente y/o investigador.

\section{Conclusiones}

A partir de los resultados obtenidos se pueden establecer las siguientes conclusiones:

- El instrumento "El semáforo" dispone de niveles de validez de contenido para evaluar la percepción del alumno sobre el nivel de aprendizaje y para evaluar la percepción del alumno sobre el grado de diversión alcanzado de iniciación en las clases de educación física.

- El instrumento diseñado, por ser rápido y de fácil cumplimiento, es un instrumento apropiado para ser utilizado en el ámbito educativo reglado.

\section{Referencias}

B.O.E. (2000). Real Decreto 1631/2000, de 28 de enero, por el que se crea la Comisión Nacional para la Protección de la Salud del Deportista.

Bulger, S. M., y Housner, L. D. (2007). Modified Delphi investigation of exercise science in physical education teacher education. Journal of Teaching in Physical Education, 26, 57-80.

Carretero-Dios, H., y Pérez, C. (2007). Normas para el desarrollo y revisión de estudios instrumentales: consideraciones sobre la selección de

test en la investigación psicológica. International Journal of Clinical and Health Psychology, 7(3), 863-882.

Casimiro, A. J. (1999). Comparación, evolución y relación de hábitos saludable, y condición física-salud en escolares entre final de educación primaria (12 ańos) y final de educación secundaria (16 años). Tesis doctoral. Universidad de Granada.

Castejón, F.J., Giménez, F.J., Jiménez, F., y López, V. (2003). Iniciación 
deportiva. La enseñanza y el aprendizaje comprensivo en el deporte. Sevilla: Wanceulen.

Cervelló, E. M; Jiménez, R; Del Villar, F.; Ramos, L. A., y Santos-Rosa, F. J. (2004). Goals orientations, motivational climate, equality, and discipline in Spanish physical education student. Perceptual and Motor Skills, 99, 271-283.

Delgado-Nogueras, M. A. (2001). La evaluación de la educación física, iyo te evalúo?, ¡tú me evalúas?, ¿antonimia didáctica? En Nuevas perspectivas de investigación en las ciencias del deporte. Cáceres: Servicio de Publicaciones de la Universidad de Extremadura.

Duda, J. (2001). Achievement goal research in sport: pushing the boundaries and clarifying some misunderstandings. In G Roberts (Ed.): $A d$ vances in motivation in sport and exercises (pp. 129-182). Champaign: Human Kinetics.

Dunn, J. G., Bouffard, M., y Rogers, W. T. (1999). Assessing Item ContentRelevance in Sport Psychology Scale-Construction Research: Issues and Recommendations. Measurement in Physical Education and Exercise Science, 3(1), 15-36.

Hassandra, M., Goudas, M., y Chroni, S. (2003). Examining factors associated with intrinsic motivation in physical education: a qualitative approach. Psychology of Sport and Exercise, 4, 211-223.

Hernández, E., Nortes, M. A., Leante, P., y Palao, J. M. (2009). Efecto de tres estrategias de enseńanza en la práctica sobre el aprendizaje de tres gestos de voleibol y la percepción del proceso en alumnos de secundaria. Lecturas: educación física y deportes, 131, 1-13.

Hernández, R., Fernández, C., y Baptista, P. (1997). Metodología de la Investigación. México: Mc Graw Hill.

McCaughtry, N., y Rovegno, I. (2003). Development of pedagogical content knowledge: moving from blaming students to predicting skilfulness, recognizing motor development, and understanding emotion. Journal of Teaching in Physical education, 22, 355-368.

McGrath, P.A., De Veber, L.L. y Hearn, M.T. (1985). Multidimensional pain assesment in children. En Fields HL, Dubner R, Cervero F, eds. Advances in pain research and therapy: proceedings from the 4th World Congress on Pain.

New York: Raven Press, 9, 387-393. Moreno, J. A., Vera, J.A., y Cervelló, E. (2006). Evaluación participativa y responsabilidad en Educación Física. Revista de Educación, 340, 731-754.

Ntounamis, N., Pensgard, A. M., Martín, C., y Pipe, K. (2004). An idiographic Analysis of Amotivation in Compulsory School Physical Edu- cation. Journal of Sport \& Exercise Psychology, 26, 197-214.

Ortega, E., Calderón, A. Palao, J. M., y Puigcerver, C. (2008). Diseńo y validación de un cuestionario para evaluar la actitud percibida del profesor en clase y de un cuestionario para evaluar los contenidos actitudinales de los alumnos durante las clases de educación física en secundaria. Retos, 14, 22-29.

Ortega, E., Jiménez, J. M., Palao, J. M., y Sainz de Baranda, M. P. (2008). Diseño y validación de un cuestionario para valorar las preferencias y satisfacciones en jóvenes jugadores de baloncesto. Cuadernos de Psicología del Deporte, 8(2), 39-58.

Padilla, J. L., Gómez, J., Hidalgo, M. D., y Muñiz, J. (2007). Esquema conceptual y procedimientos para analizar la validez de las consecuencias del uso de los test. Psicothema,19(19), 173-178.

Palao, J. M. y Ruiz, E. (2003). Percepción de los alumnos universitarios del efecto de la modificación de variables en las formas jugadas en la iniciación al voleibol. En J.P. Fuentes y M. Bellido (Ed.), Actas del I Congreso Europeo FIEP (pp. 499-508). Diputación de Cáceres: Cáceres.

Sallis, J. F., Mckenzie, T. L., y Alcaráz, F. E. (1993). Habitual Physical activity and Elath-related physical fitness in fourth-grade children. American Journal of deseases in childhood, 147, 890-896.

Siendentop, D. (1998). Aprender a enseñar en educación física. Barcelona: Inde.

Silverman, S. (1985). Relationship of engagement and practice trials to student achievement. Journal and Teaching Physical Education, 5, 13-21.

Silverman, S., y Subramanian, P.R. (1999). Student attitude toward education and physical activity: a review of measurement issues and outcomes. Journal of Teaching in Physical Education, 19, 97-125.

Subramanian, P. R., y Silverman, S. (2000). Validation of scores from an instrument assessing student attitude toward physical education. Measurement in Physical Education \& Exercise Science, 4(1), 29-43.

Thomas, J. R., y Nelson, J. K. (2007). Métodos de investigación en actividad física. Barcelona: Paidotribo.

Weinberg, R. S., y Gould, D. (1995). Foundations of sport and exercise psychology. Champaign: Human Kinetcs.

Wiersma, L. D. (2001). Conceptualization and development of the sources of enjoyment in youth sport questionnaire. Measurement in Physical Education and Exercise Science, 5(3), 153-177.

Xiang, P., McBridge, R., y Guan, J. (2004). Children 's motivation in elementary physical education: A longitudinal study. Research Quaterly for Exercise and Sport, 75(1), 71-80.

\section{Anexos}

Versión inicial del instrumento para valorar la percepción del aprendizaje en las clases de educación física en alumnos de la Etapa de Educación Secundaria Obligatoria (anexo 1). 


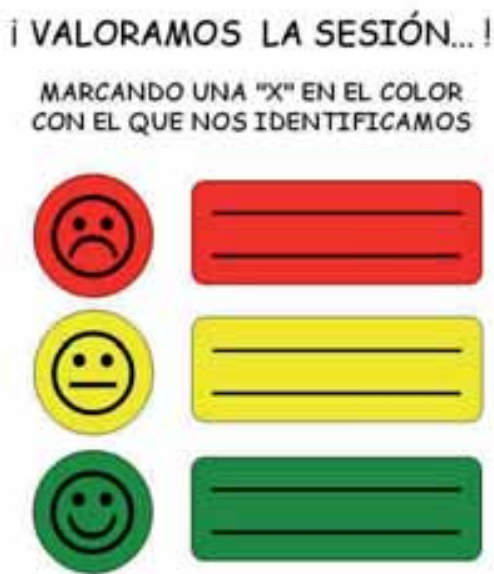

i... PARA MEJORAR!

¿Cómo te ha parecido la sesión a nivel de aprendizaje?

Versión inicial del instrumento para valorar el nivel de diver-

Educación Secundaria Obligatoria (anexo 2).

sión en clases de educación física en alumnos de la Etapa de

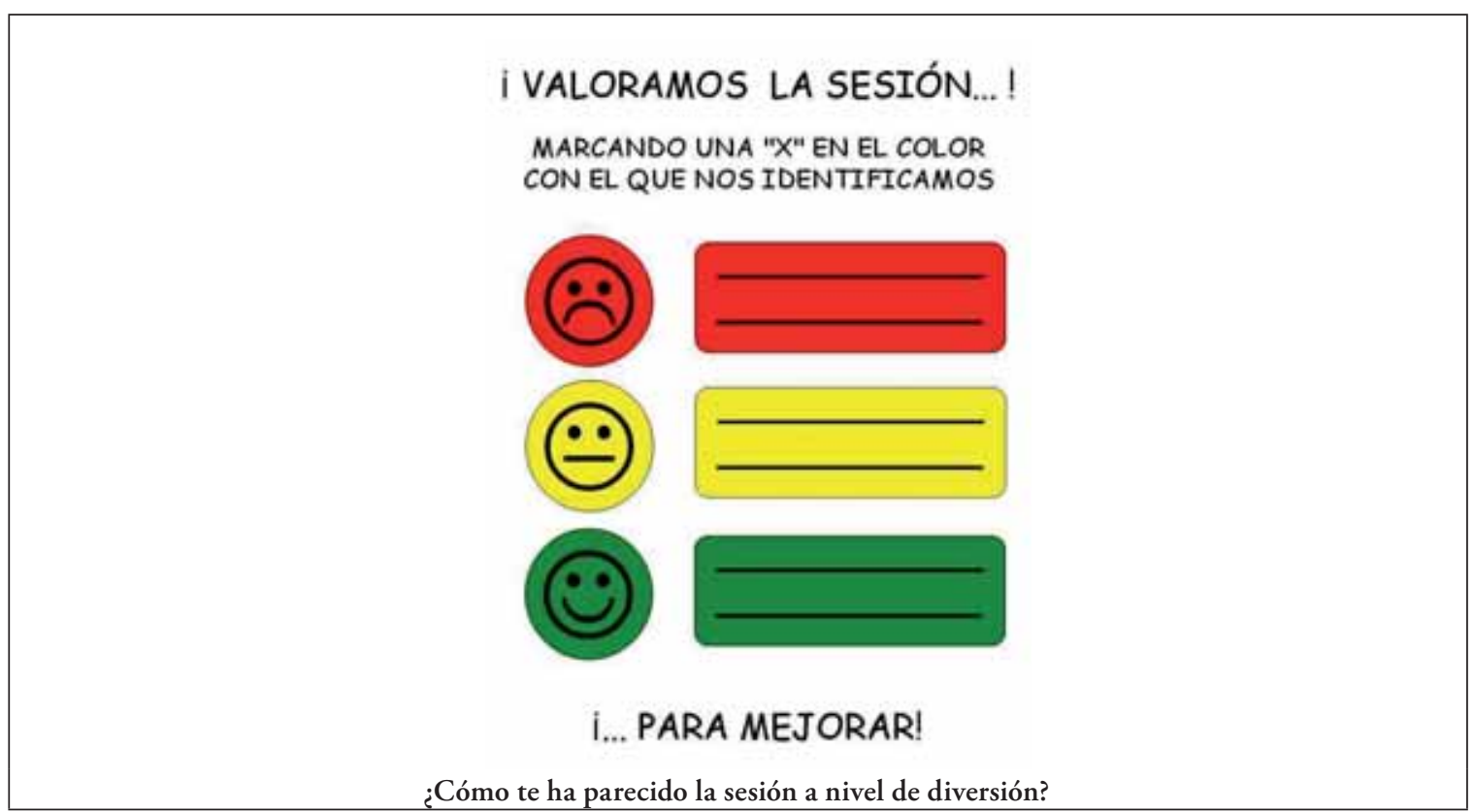

Versión definitiva del instrumento para valorar la percepción del aprendizaje en las clases de educación física en alumnos de la Etapa de Educación Secundaria Obligatoria (anexo 3). 


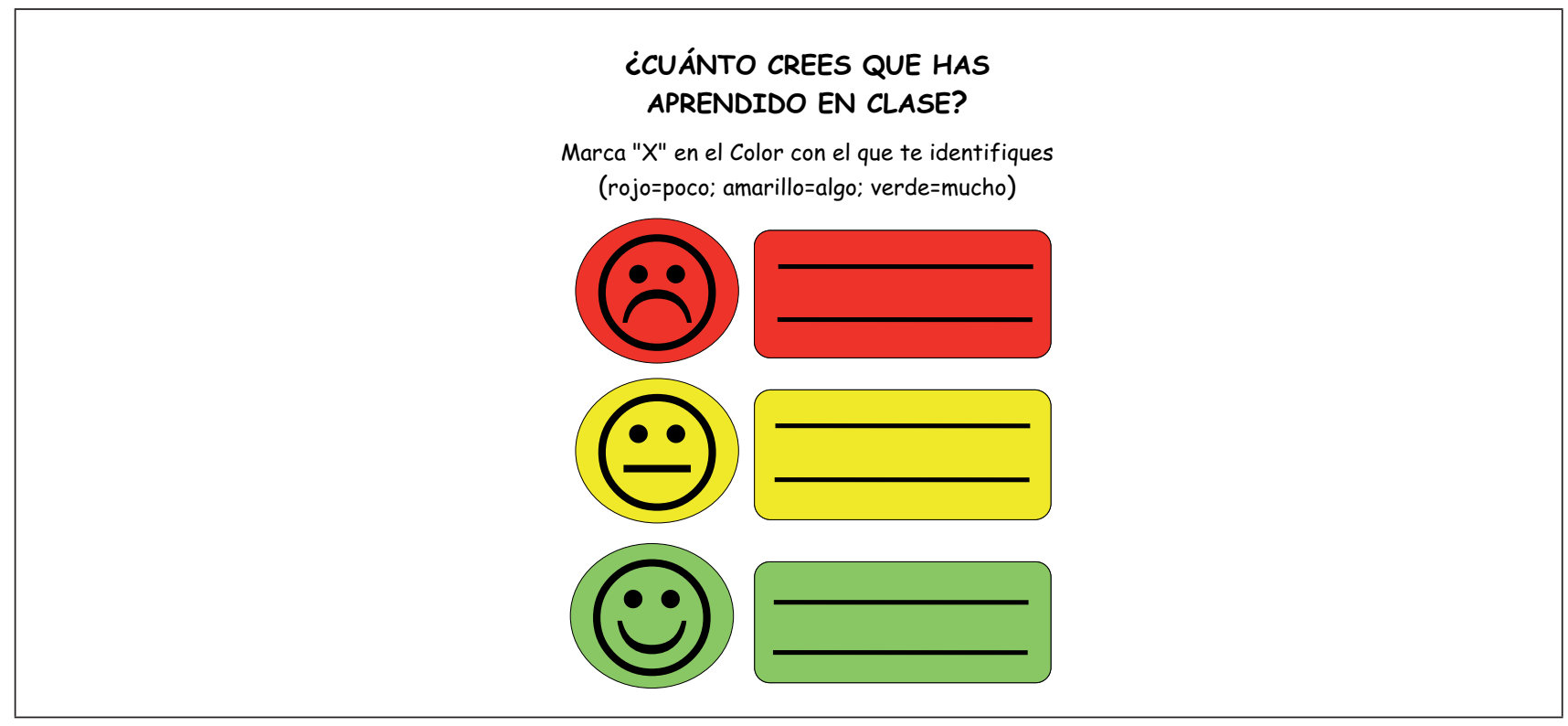

Versión definitiva del instrumento para valorar el nivel de diversión en las clases de educación física en alumnos de la Etapa de Educación Secundaria Obligatoria (anexo 4).

\section{¿CUÁNTO TE HAS \\ DIVERTIDO EN CLASE?}

Marca " $X "$ en el Color con el que te identifiques

(rojo=poco; amarillo=algo; verde=mucho)
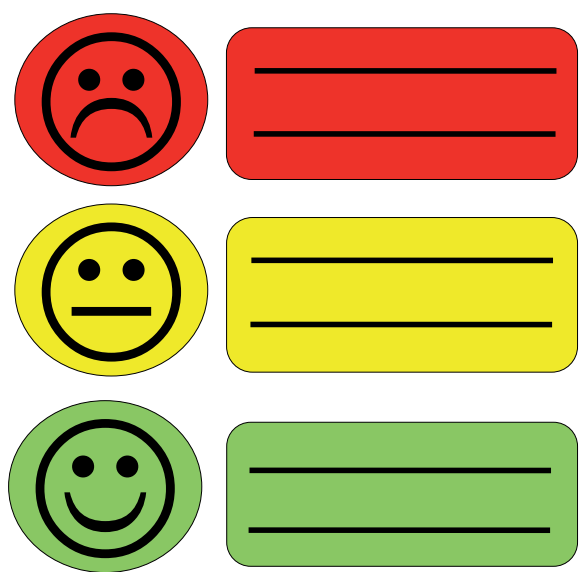